\title{
Multilocus microsatellite typing of Leishmania infantum isolates in monitored Leishmania/HIV coinfected patients

Míriam Tomás-Pérez ${ }^{1}$, Mallorie Hide², Cristina Riera ${ }^{1}$, Liliana Montoya ${ }^{1}$, Anne-Laure Bañuls ${ }^{2}$, Esteve Ribera ${ }^{3}$, Montserrat Portús ${ }^{1}$ and Roser Fisa ${ }^{1 *}$

\begin{abstract}
Background: Leishmania infantum is the main etiological agent of both visceral and cutaneous clinical forms of leishmaniasis in the Mediterranean area. Leishmania/HIV coinfection in this area is characterized by a chronic course and frequent recurrences of clinical episodes. The present study using Multilocus Microsatellite Typing (MLMT) analysis, a highly discriminative tool, aimed to genetically characterize $L$. infantum isolates taken from monitored Leishmania/HIV coinfected patients presenting successive clinical episodes.

Methods: In this study, by the analysis of 20 microsatellite loci, we studied the MLMT profiles of $25 \mathrm{~L}$. infantum isolates from 8 Leishmania/HIV coinfected patients who had experienced several clinical episodes. Two to seven isolates per patient were taken before and after treatment, during clinical and non-clinical episodes, with time intervals of 6 days to 29 months. Genetic diversity, clustering and phenetic analyses were performed.

Results: MLMT enabled us to study the genetic characteristics of the $25 \mathrm{~L}$. infantum isolates, differentiating 18 genotypes, corresponding to a genotypic diversity of 0.72 . Fifteen genotypes were unique in the total sample set and only 3 were repeated, 2 of which were detected in different patients. Both clustering and phylogenetic analyses provided insights into the genetic links between the isolates; in five patients isolates showed clear genetic links: either the genotype was exactly the same or only slightly different. In contrast, the isolates of the other three patients were dispersed in different clusters and some could be the result of mixing between populations.

Conclusions: Our data indicated a great MLMT variability between isolates from coinfected patients and no predominant genotype was observed. Despite this, almost all clinical episodes could be interpreted as a relapse rather than a reinfection. The results showed that diverse factors like an intrapatient evolution over time or culture bias could influence the parasite population detected in the patient, making it difficult to differentiate between relapse and reinfection.
\end{abstract}

Keywords: Leishmania infantum, HIV+, Microsatellites, Genetic diversity, Phenetic analyses, Relapse, Reinfection

\section{Background}

The first case of leishmaniasis associated with HIV infection was reported in 1985. Since then, cases of coinfection have been reported in 35 countries around the world, with an increasing number occurring in southern Europe. The four European countries most affected are France, Italy, Portugal and above all Spain, the latter

\footnotetext{
*Correspondence: rfisa@ub.edu

'Laboratory of Parasitology, Faculty of Pharmacy, Universitat de Barcelona, Avda Joan XXIII s/n, 08028 Barcelona, Spain

Full list of author information is available at the end of the article
}

having the highest incidence due to a greater geographical overlap between leishmaniasis and HIV infections [1]. Leishmania infantum is the main etiological agent of both visceral and cutaneous clinical forms of human leishmaniasis in this area, which is transmitted through vectors of the species Phlebotomus perniciosus and P. ariasi [2]. These cases of leishmaniasis/HIV coinfection usually result in visceral leishmaniasis (VL), and are often thought to be related to the reactivation of asymptomatic infections [1]. Acquired infection among intravenous drug users (IVDU) sharing contaminated syringes has also been

\section{() Biomed Central}


reported, which could represent an anthroponotic cycle [3]. VL cases associated with HIV are characterized by the appearance of numerous recurrences of clinical episodes that vary in number and average duration according to the immunological status of patients, parasite species, and use of anti-leishmanial therapy [1].

For the epidemiological study and follow-up of these coinfection cases, highly discriminatory methods are needed to differentiate Leishmania at the strain level $[4,5]$. Previous research on different Leishmania strains of coinfected patients in France using isoenzymatic characterization (MLEE) revealed a low polymorphism in primo infection as well as in relapse cases [6]. Research performed in Catalonia, our area of study, has also registered a low polymorphism among coinfected patients, with more than $50 \%$ of the studied strains belonging to zymodeme MON-1 [7]; in the south of Spain, however, a greater polymorphism was found by MLEE studies, although almost $50 \%$ of strains were described as zymodeme MON-1 $[8,9]$.

Microsatellite markers have proved to be powerful tools for molecular typing and population genetic studies in Leishmania, being able to discriminate among zymodemes, even within MON-1 [4]. Microsatellites are short nucleotide fragments of 1 to $6 \mathrm{bp}$ repeated in tandem and ubiquitously distributed in the genomes of eukaryotic organisms [10]. They present high rates of mutation and variability due to allelic repeat length variation [11, 12]. The Multilocus Microsatellite Typing (MLMT) approach developed for the Leishmania donovani complex is based on a set of 14-20 unlinked microsatellite loci [12]. The present MLMT study aimed to genetically characterize $L$. infantum isolates taken from monitored Leishmania/HIV coinfected patients presenting subsequent clinical episodes. The specific objective was to define the genetic links between the parasites of VL patients under study, and between parasites from repeated isolates from the same patient. Besides describing a Leishmania population in HIV patients, this genetic study attempted to generate data that would help to differentiate between parasite reinfections and relapses.

\section{Methods}

\section{Patients}

The study included 8 adults ( 7 men and 1 woman) with Leishmania/HIV coinfection from the Barcelona metropolitan area, monitored at the Hospital Vall d'Hebron (Barcelona, Spain). Six patients were IVDU, aged from 27 to 44 years at the start of the study, when the first Leishmania isolate was obtained (Table 1).

All patients were under HAART therapy (Highly Active AntiRetroviral Therapy). Diagnosis of VL was confirmed by culture in bone marrow or peripheral blood. After diagnosis, patients received one of the following treatments at standard doses: liposomal amphotericin B or amphotericin B lipid complex.

\section{Ethical approval}

The study protocol was approved by the institutional review board of the hospital, and all patients signed informed consent for their participation in this study.

\section{Leishmania isolates}

Twenty-five isolates were obtained from clinical and non-clinical episodes of the 8 patients. Two to seven isolates per patient were taken before and after treatment, with time intervals of 6 days to 29 months (Table 1). Eighteen isolates were obtained during clinical episodes and seven during non-clinical episodes. "In vitro" culture was performed using NNN (Novy-McNeal-Nicolle's) medium and/or Schneider's insect culture medium (Sigma, St. Louis, MO) supplemented with $20 \%$ heatinactivated fetal calf serum, $1 \%$ sterile human urine, and $25 \mu \mathrm{g} / \mathrm{mL}$ gentamicin solution. Cultures were maintained between $24{ }^{\circ} \mathrm{C}$ and $26{ }^{\circ} \mathrm{C}$, examined twice a week, and sub-cultured every 2 weeks for 6 months before being considered negative. All isolates were stored in the Leishmania Cryobank at the Universitat de Barcelona. A L. infantum strain from Toulouse (France) was used as a reference to build the neighbor-joining (NJ) tree: LEM2355 (WHO code: MHOM/FR/91/LEM2355; MON-183).

\section{DNA extraction}

One cryovial from each isolate was used for isolation of DNA. The promastigotes were quickly thawed and DNA was extracted using a chelex resin protocol: $100 \mu \mathrm{L}$ of sterile water and $400 \mu \mathrm{L}$ of chelex solution [1\% Tween 20 (Sigma, St. Louis, MO), 1 \% Nonidet P-40 (Sigma, St. Louis, MO) and $20 \%$ of chelex resin (BioRad Laboratories, Hercules, CA)] were added to the promastigote sediment. It was heated at $100{ }^{\circ} \mathrm{C}$ for $20 \mathrm{~min}$ and then vortexed. Finally, the mixture was centrifuged for $10 \mathrm{~min}$ at $12,000 \mathrm{~g}$ to separate the resin and the supernatant was collected as the substrate for the PCR, either performed immediately or after storage at $-20{ }^{\circ} \mathrm{C}$. All isolates were confirmed as $L$. infantum by specific PCR $[13,14]$.

\section{Multilocus Microsatellite Typing (MLMT)}

The genotyping was done using 20 microsatellite markers previously described for the genetic characterization of L. infantum (Table 2). Amplification was performed in a volume of $30 \mu \mathrm{L}$ containing $3 \mu \mathrm{L}$ of $10 \mathrm{X}$ buffer, $1 \mathrm{nmol}$ of dNTP mix, and $10 \mathrm{pmol}$ of each primer (the forward being labeled) and 1.5 units of Taq polymerase (Taq Polymerase, $5 \mathrm{U} / \mu \mathrm{L}$, Roche Diagnostics, France). 
Table 1 Identification of the 25 Leishmania isolates used in this study from 8 Leishmania/HIV coinfected patients obtained during $\mathrm{VL}$ clinical episodes or non-clinical episodes, from bone marrow or peripheral blood mononuclear cell samples; and their genotyping and clustering analysis results

\begin{tabular}{|c|c|c|c|c|c|c|c|c|c|c|}
\hline $\begin{array}{l}\text { Patient } \\
\text { code }\end{array}$ & $\begin{array}{l}\text { Patient } \\
\text { isolates }\end{array}$ & Sex & Age & $\begin{array}{l}\text { Risk } \\
\text { factor }\end{array}$ & $\begin{array}{l}\text { Time from first } \\
\text { episode (in months) }\end{array}$ & $\begin{array}{l}\text { Clinical } \\
\text { status }\end{array}$ & $\begin{array}{l}\text { Sample } \\
\text { Type }\end{array}$ & WHO Code & $\begin{array}{l}\text { Genotype } \\
\text { (G) }\end{array}$ & $\begin{array}{l}\text { Cluster } \\
\text { (C) }\end{array}$ \\
\hline \multirow[t]{3}{*}{$\mathrm{P1}$} & $1 a$ & Man & 30 & IVDU & June & CE & PBMC & $\begin{array}{l}\text { MHOM/ES/00/BCN- } \\
278\end{array}$ & G14 & $\mathrm{c} 2 \mathrm{~b}$ \\
\hline & $1 b$ & & & & $8 \mathrm{~m}$ & CE & PBMC & $\begin{array}{l}\text { MHOM/ES/01/BCN- } \\
376\end{array}$ & G13 & $\mathrm{c} 2 \mathrm{~b}$ \\
\hline & $1 \mathrm{c}$ & & & & $20 \mathrm{~m}$ & CE & PBMC & $\begin{array}{l}\text { MHOM/ES/02/BCN- } \\
464\end{array}$ & G15 & $\mathrm{C} 2 \mathrm{~b}$ \\
\hline \multirow[t]{2}{*}{ P2 } & $2 a$ & Man & 44 & IVDU & July & CE & $\mathrm{BM}$ & $\begin{array}{l}\mathrm{MHOM} / \mathrm{ES} / 00 / \mathrm{BCN}- \\
284\end{array}$ & G3 & $\mathrm{C} 1$ \\
\hline & $2 b$ & & & & $2 \mathrm{~m}$ & CE & $\mathrm{BM}$ & $\begin{array}{l}\text { MHOM/ES/O0/BCN- } \\
289\end{array}$ & G2 & $\mathrm{C} 1$ \\
\hline \multirow[t]{3}{*}{ P3 } & $3 a$ & Man & 36 & nd & May & CE & BM & $\begin{array}{l}\text { MHOM/ES/01/BCN- } \\
404\end{array}$ & G16 & $\mathrm{C} 2$ \\
\hline & $3 b$ & & & & $7 \mathrm{~m}$ & $\mathrm{NE}$ & PBMC & $\begin{array}{l}\text { MHOM/ES/01/BCN- } \\
455\end{array}$ & G16 & $\mathrm{C} 2$ \\
\hline & $3 c$ & & & & $12 \mathrm{~m}$ & CE & BM & $\begin{array}{l}\text { MHOM/ES/02/BCN- } \\
492\end{array}$ & G16 & $\mathrm{C} 2$ \\
\hline \multirow[t]{2}{*}{ P4 } & $4 a$ & Man & 34 & IVDU & April & CE & BM & $\begin{array}{l}\text { MHOM/ES/02/BCN- } \\
475\end{array}$ & G9 & $\mathrm{C} 2$ \\
\hline & $4 b$ & & & & $6 \mathrm{~m}$ & CE & $\mathrm{BM}$ & $\begin{array}{l}\text { MHOM/ES/02/BCN- } \\
508\end{array}$ & G9 & $\mathrm{C} 2$ \\
\hline \multirow[t]{2}{*}{ P5 } & $5 a$ & Man & 36 & IVDU & September & CE & $\mathrm{BM}$ & $\begin{array}{l}\text { MHOM/ES/94/BCN- } \\
123\end{array}$ & G10 & $\mathrm{C} 2$ \\
\hline & $5 b$ & & & & $5 \mathrm{~m}$ & CE & BM & $\begin{array}{l}\text { MHOM/ES/95/BCN- } \\
130\end{array}$ & G1 & - \\
\hline \multirow[t]{3}{*}{ P6 } & $6 a$ & Man & 33 & IVDU & October & CE & BM & $\begin{array}{l}\text { MHOM/ES/O0/BCN- } \\
298\end{array}$ & G11 & $\mathrm{C} 2$ \\
\hline & $6 b$ & & & & $6 \mathrm{~m}$ & CE & BM & $\begin{array}{l}\text { MHOM/ES/01/BCN- } \\
400\end{array}$ & G5 & $\mathrm{C} 2$ \\
\hline & $6 c$ & & & & $17 \mathrm{~m}$ & CE & $\mathrm{BM}$ & $\begin{array}{l}\text { MHOM/ES/02/BCN- } \\
470\end{array}$ & G12 & $C 2$ \\
\hline \multirow[t]{3}{*}{ P7 } & $7 a$ & Man & 41 & nd & October & CE & PBMC & $\begin{array}{l}\text { MHOM/ES/01/BCN- } \\
430\end{array}$ & G7 & $\mathrm{C} 2 \mathrm{a}$ \\
\hline & $7 b$ & & & & $3 \mathrm{~m}$ & $\mathrm{NE}$ & $\mathrm{BM}$ & $\begin{array}{l}\text { MHOM/ES/02/BCN- } \\
460\end{array}$ & G7 & $\mathrm{C} 2 \mathrm{a}$ \\
\hline & $7 c$ & & & & $4 \mathrm{~m}$ & CE & PBMC & $\begin{array}{l}\text { MHOM/ES/02/BCN- } \\
472\end{array}$ & G6 & $\mathrm{C} 2 \mathrm{a}$ \\
\hline \multirow[t]{7}{*}{ P8 } & $8 a$ & Woman & 27 & IVDU & October & CE & PBMC & $\begin{array}{l}\text { MHOM/ES/O0/BCN- } \\
293\end{array}$ & G4 & $C 2$ \\
\hline & $8 b$ & & & & $1 \mathrm{~m}$ & $\mathrm{NE}$ & PBMC & $\begin{array}{l}\text { MHOM/ES/O0/BCN- } \\
306\end{array}$ & G7 & $\mathrm{C} 2 \mathrm{a}$ \\
\hline & $8 c$ & & & & $1 m+6$ days & $\mathrm{NE}$ & PBMC & $\begin{array}{l}\text { MHOM/ES/01/BCN- } \\
307\end{array}$ & G8 & $\mathrm{C} 2$ \\
\hline & $8 d$ & & & & $3 \mathrm{~m}$ & $\mathrm{NE}$ & PBMC & $\begin{array}{l}\text { MHOM/ES/01/BCN- } \\
369\end{array}$ & G16 & $\mathrm{C} 2$ \\
\hline & $8 e$ & & & & $7 \mathrm{~m}$ & CE & PBMC & $\begin{array}{l}\text { MHOM/ES/01/BCN- } \\
405\end{array}$ & G18 & $\mathrm{C} 2$ \\
\hline & $8 f$ & & & & $9 \mathrm{~m}$ & NE & PBMC & $\begin{array}{l}\text { MHOM/ES/01/BCN- } \\
422\end{array}$ & G17 & $\mathrm{C} 2$ \\
\hline & $8 \mathrm{~g}$ & & & & $29 \mathrm{~m}$ & $\mathrm{NE}$ & PBMC & $\begin{array}{l}\text { MHOM/ES/03/BCN- } \\
561\end{array}$ & G16 & $\mathrm{C} 2$ \\
\hline
\end{tabular}


Table 2 Characteristics of the 20 microsatellite loci used in this study for Leishmania infantum genotyping

\begin{tabular}{|c|c|c|c|c|c|c|c|}
\hline Locus & GenBank accession no. & Allele size (bp) & Dye label & $\mathrm{Ta}\left({ }^{\circ} \mathrm{C}\right)$ & $\mathrm{Na}$ & $H_{S}$ & $H_{0}$ \\
\hline$\overline{L i 22-35^{c}}$ & AM050045 & 90-106 & VIC & 58 & 5 & 0.191 & 0.042 \\
\hline Li45-24c & AM050048 & $88-108$ & NED & 58 & 5 & 0.208 & 0.060 \\
\hline $\mathrm{Li} 71-5 / 2^{c}$ & AM050050 & 104-108 & $\mathrm{VIC}$ & 54 & 3 & 0.280 & 0.042 \\
\hline $\mathrm{Li} 72-20^{c}$ & AM050057 & $87-95$ & VIC & 50 & 4 & 0.275 & 0.042 \\
\hline LiBTA $^{a}$ & nd & $226-246$ & $\mathrm{VIC}$ & 58 & 4 & 0.316 & 0.167 \\
\hline $\mathrm{LiBTG}^{\mathrm{a}}$ & nd & $219-257$ & 6-FAM & 58 & 7 & 0.340 & 0.104 \\
\hline LIST7021 ${ }^{\text {b }}$ & AF427869 & $228-246$ & 6-FAM & 54 & 6 & 0.338 & 0.143 \\
\hline LIST7024 & AF427872 & $198-224$ & $\mathrm{VIC}$ & 59 & 3 & 0.049 & 0.000 \\
\hline LIST7025 & AF427873 & $171-179$ & 6-FAM & 56 & 1 & 0.000 & 0.000 \\
\hline LIST7026 & AF427874 & $201-231$ & NED & 56 & 4 & 0.089 & 0.000 \\
\hline LIST7028 & AF427876 & 104-108 & $\mathrm{VIC}$ & 58 & 2 & 0.049 & 0.000 \\
\hline LIST7031 $^{\mathrm{b}}$ & AF427879 & $166-174$ & PET & 54 & 1 & 0.000 & 0.000 \\
\hline LIST7033 & AF427881 & $196-226$ & 6-FAM & 58 & 5 & 0.349 & 0.411 \\
\hline LIST7035 b & AF427883 & 188-202 & PET & 56 & 6 & 0.373 & 0.146 \\
\hline LIST7037 & AF427885 & 178-194 & 6-FAM & 58 & 5 & 0.320 & 0.411 \\
\hline LIST7038 & AF427886 & $122-130$ & NED & 56 & 3 & 0.088 & 0.000 \\
\hline LIST7039 & AF427887 & 199-215 & PET & 58 & 5 & 0.128 & 0.143 \\
\hline Rossi1 ${ }^{d}$ & X76394 & $104-110$ & 6-FAM & 59 & 3 & 0.100 & 0.000 \\
\hline Rossi2 ${ }^{d}$ & X76393 & $140-160$ & VIC & 57 & 6 & 0.335 & 0.185 \\
\hline TubCA & nd & $74-84$ & 6-FAM & 58 & 2 & 0.100 & 0.000 \\
\hline Mean value & & & & & 4 & 0.196 & 0.095 \\
\hline
\end{tabular}

Ta: annealing temperature (thermocycling conditions); Na: number of alleles; Hs: Nei's unbiased genetic diversity within subsamples; Ho: observed heterozygosity

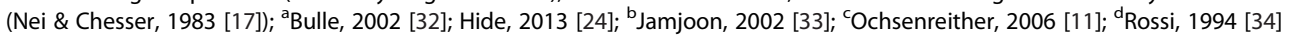

We added $50 \mathrm{ng}$ of extracted DNA to the mixture and incubated it in a thermal cycler under the following conditions: a denaturing step at $94{ }^{\circ} \mathrm{C}$ for $2 \mathrm{~min}$, followed by 35 cycles of denaturation for $30 \mathrm{~s}$ at $94{ }^{\circ} \mathrm{C}$, annealing for $1 \mathrm{~min}$ at the annealing temperature of each locus (Table 2) and extension for $1 \mathrm{~min}$ at $72{ }^{\circ} \mathrm{C}$, followed by a final extension at $72{ }^{\circ} \mathrm{C}$ for $30 \mathrm{~min}$. The amplified products were analyzed using an automated fragment analysis on an ABI Prism 3130XL genetic analyzer (Applied Biosystems, France) with a Genescan 500 LIZ internal size standard. Finally, data were analyzed with GeneMapper analysis software (version 4.0, Applied Biosystems, France).

\section{Genetic diversity and differentiation analysis}

Data were analyzed with FSTAT Version 2.9.3.2 [15] updated from [16], which allows the calculation of diversity indices such as Nei's unbiased genetic diversity index within sub-samples $(H \mathrm{~s})$, the observed heterozygosity (Ho), as a measure of genetic diversity [17], and the number of alleles per locus (Na) measuring genetic polymorphism. The genotypic diversity was calculated as the ratio of the number of genotypes per total number of samples.

\section{Clustering and phenetic analyses}

The genetic characteristics of the Leishmania samples under study were investigated with MLMT data by two different methods. The first was based on genetic distances by the construction of a phenetic tree according to the proportion of shared allele distances $\left(\mathrm{D}_{\mathrm{AS}}\right)$. The Neighbor-Joining $(\mathrm{NJ})$ tree [18] was constructed through calculations of Cavalli-Sforza genetic distance from allelic frequencies, and the robustness of tree topology was obtained by bootstrap resampling of loci, with 100 replications per set. We used PHYLIP software (3.67 package; J. Felsenstein, 1993. Department of Genetics, University of Washington, Seattle, USA) and the tree was edited and visualized with TreeDyn software [19]. The second approach consisted of a model-based Bayesian clustering method implemented in STRUCTURE v 2.3.1 [20]. This algorithm simultaneously estimates the allele frequencies to assign individuals into genetically distinct populations $(K)$ and each probability for the identification of the most likely number of populations. The allele frequencies among populations were correlated by admixture modeling for a series of runs using a 'burn-in' period of 20,000 iterations and probability estimates based on 200,000 of Markov chain Monte Carlo (MCMC) repeats. 
Ten independent runs for each $K$ were carried out for each possible number of clusters $(K)$ in order to quantify the variation in the likelihood of the data for a given $K$. The most appropriate number of populations was determined based upon ad hoc statistic $\Delta K$, which evaluates the second order rate of change of the likelihood function with respect to the number of populations $(K)$.

\section{Results}

\section{Genetic diversity}

All 20 microsatellite loci used for the analysis of the 25 L. infantum isolates produced clear electrophoregrams, with only one or two alleles at each locus. We found 18 polymorphic microsatellite markers, with LiBTG as the most polymorphic, having 7 different alleles $\left(N_{\mathrm{a}}\right)$ and two monomorphic markers, LIST7025 and LIST7031. We observed 8 polymorphic microsatellites with more than 5 alleles, LIST7021, LIST7035 and Rossi2 with 6 alleles each; and Li22-35, Li45-24, LIST7033, LIST7037 and LIST7039 with 5 alleles. Markers Li72-20, LiBTA and LIST7026 revealed 4 alleles; Li71-5/2, LIST7024, Rossi1 and LIST7038 3 alleles; TubCA and LIST7028 showed 2 alleles. The mean value was 4 alleles per locus (Table 2). The diversity analysis, including all the 25 isolates, revealed an observed heterozygosity $\left(H_{\mathrm{o}}\right)$ between 0 and 0.411 (overall 0.095); the mean intrapopulation genetic diversity $\left(H_{\mathrm{s}}\right)$ ranged between 0 and 0.373 , with an overall value of 0.196 (Table 2).

\section{Genotype analysis and phylogenetic reconstruction}

A total of 18 genotypes $(G)$ were characterized for the 25 isolates belonging to the 8 monitored patients (Table 1), corresponding to a genotypic diversity of 0.72 . Of these genotypes, 15 were unique in the total sample set, and only 3 (G7, G9 and G16) were repeated. The three repeated genotypes were found in four different patients: G7 in two of the three isolates of patient P7 and one of the seven isolates of patient P8; G9 in the two isolates of patient P4; and G16 in the three isolates of patient P3 and two isolates of patient P8. Only genotypes G16 and G7 were detected in two different patients.

MLMT profiles were used to calculate genetic distances and build a neighbor-joining tree. The genetic distance tree allowed us to differentiate 2 main populations or clusters $\mathrm{C} 1$ and $\mathrm{C} 2$, the latter composed of 2 well-supported sub-clusters $\mathrm{C} 2 \mathrm{a}$ and $\mathrm{C} 2 \mathrm{~b}$ (Fig. 1). The reference strain LEM2355 was used as the outgroup. At the top of the tree, isolate $5 \mathrm{~b}$ from patient P5 is clearly separated from the others. The first cluster (C1) is composed of the two isolates obtained from patient P2, separated from the others with a strong bootstrap value of $100 \%$. The second cluster (C2) is defined by a bootstrap value of $84 \%$ and contains all the other 22 isolates. In
C2 we can detect several sub-clusters, but only two, representing 5 samples and designated as $\mathrm{C} 2 \mathrm{a}$ and $\mathrm{C} 2 \mathrm{~b}$, are supported by bootstrap values higher than $80 \%$. C2a, with a bootstrap value of $100 \%$, is composed of the three isolates of patient P7 and one isolate (8b) of patient P8; C2b is supported by a bootstrap value of $84 \%$ and includes all isolates from patient P1.

\section{Clustering analysis}

The population organization of the 25 isolates was analyzed with STRUCTURE software for a better visualization of the data. Using the methods of Evanno and Garnier [21, 22], the analysis indicated the existence of 6 different populations $(K=6)$ (Figs. 1 and 2). Considering $K=6$, all isolates from patients $\mathrm{P} 2$ and $\mathrm{P} 1$ defined two of these six populations, corresponding to the cluster $\mathrm{C} 1$ and the sub-cluster $\mathrm{C} 2 \mathrm{~b}$ of the phenetic tree. A third STRUCTURE population was defined by all isolates from patient P7 and one isolate from patient P8 (8b), corresponding to the sub-cluster C2a. A fourth population included all other isolates from $\mathrm{C} 2$, which were all isolates from patients $\mathrm{P} 3$ and $\mathrm{P} 4$, and some isolates belonging to patients P5, P6 and P8 (5a, 6a, 6c, 8c, 8d, 8e, 8f and $8 \mathrm{~g})$. The two other populations were defined by the outgroup and one isolate from patient P5 (5b). The STRUCTURE analysis did not allow the classification of two isolates, $8 \mathrm{a}$ and $6 \mathrm{~b}$, which appear as mixed genotypes of NJ tree populations, $\mathrm{C} 2, \mathrm{C} 2 \mathrm{a}$ and the nonclassified isolate $5 \mathrm{~b}$ from patient $\mathrm{P} 5$.

\section{Discussion}

L. infantum is the causative species of VL and CL in the northwest Mediterranean area and an opportunistic parasite in HIV patients. It appears that immunocompromised people may be vulnerable to parasites that either fail to survive or never cause detectable morbidity in immunocompetent people. Furthermore, "dermotropic" variants of $L$. infantum have been reported to cause visceral disease in HIV-positive patients $[1,8,23]$. Other studies have revealed differences in parasitic genotypes between L. infantum strains from asymptomatic carriers and HIV-positive VL patients, suggesting that some genotypes do not cause disease [24]. Greater knowledge about the intraspecific variability of the Leishmania parasite from HIV-positive VL patients may help to understand some important key points, such as transmission patterns, response to treatment and the importance of immunity, and the parasite's capacity to survive within human hosts.

In the present study, MLMT characterization of 25 $L$. infantum isolates showed the existence of a genetic polymorphism, with a mean number of alleles per 


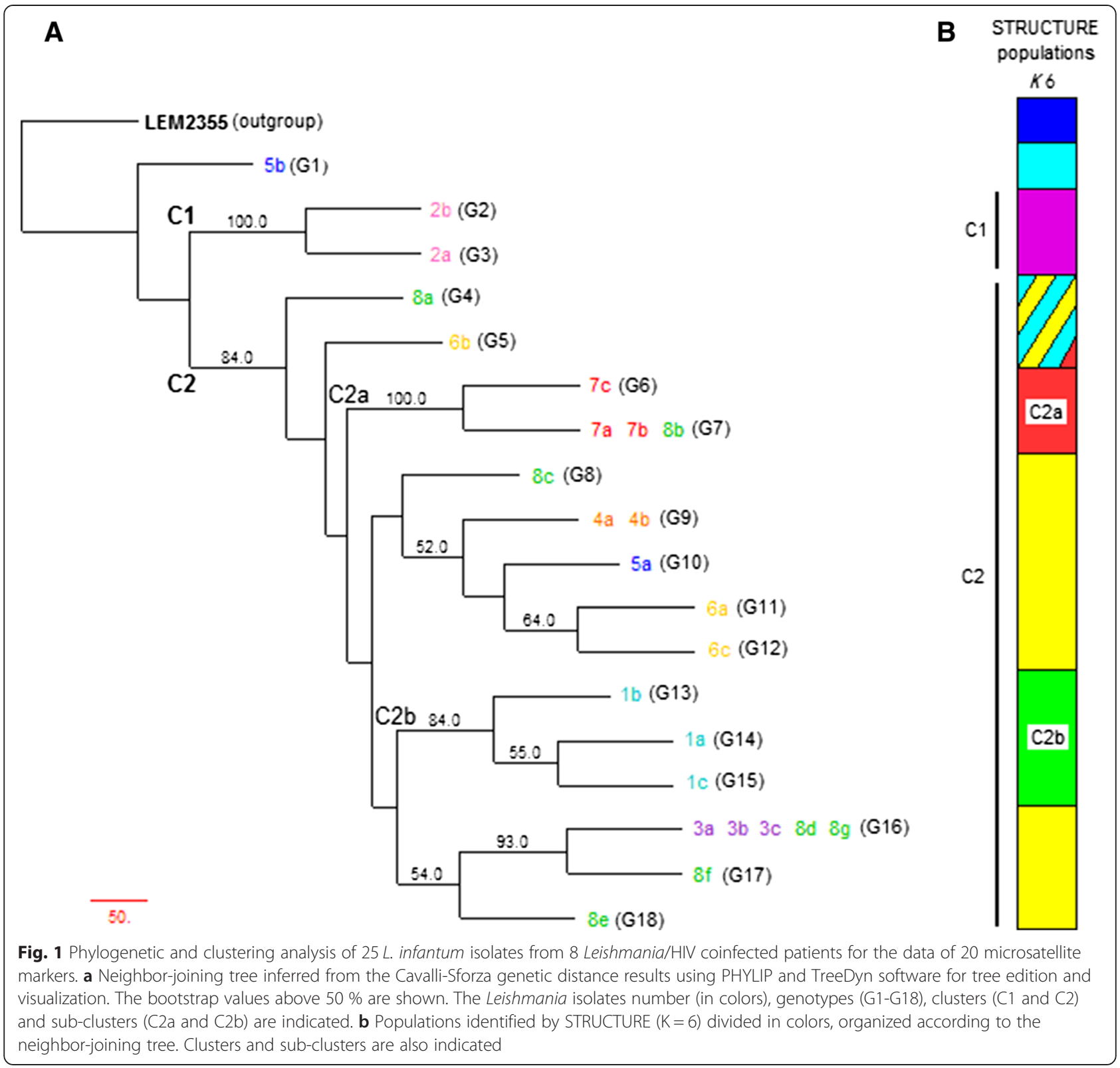

locus $\left(N_{\mathrm{a}}\right)$ of 4 . Considering that our sample set came from only 8 coinfected patients monitored over time, from the restricted geographical area of Barcelona, the $N_{\mathrm{a}}$ is not negligible when compared with previous studies performed in nearby areas. In the South of France, a mean $N_{\mathrm{a}}$ of 4.13 was found within $L$. infantum isolates from symptomatic humans and dogs and asymptomatic humans [24]. A similar mean $N_{\mathrm{a}}$ value of 4.57 was obtained in Portugal from human, dog, vulpine and phlebotomine $L$. infantum isolates [25]. Likewise, another study performed on MON-1 $L$. infantum isolates from human patients, dogs and phlebotomine sand flies of Spain, Portugal and Greece obtained a mean $N_{\mathrm{a}}$ value of 4.6 [4].
Furthermore, 18 different genotypes were found in the 25 analyzed isolates, with a low number of repeated genotypes, despite their limited geographical and human origin. Fifteen genotypes were unique in the total sample set, and only three (G7, G9 and G16) were repeated; both G7 and G16 appeared in two different patients, and G9 in two isolates from the same patient. No relationship was observed between genotypes and the clinical status of patients, with 16 different genotypes among the 18 clinical episodes, and two (G7 and G16) of the three repeated genotypes present in clinical and non-clinical episodes. The kind of sample used in the study, bone marrow or peripheral blood, was not associated with 


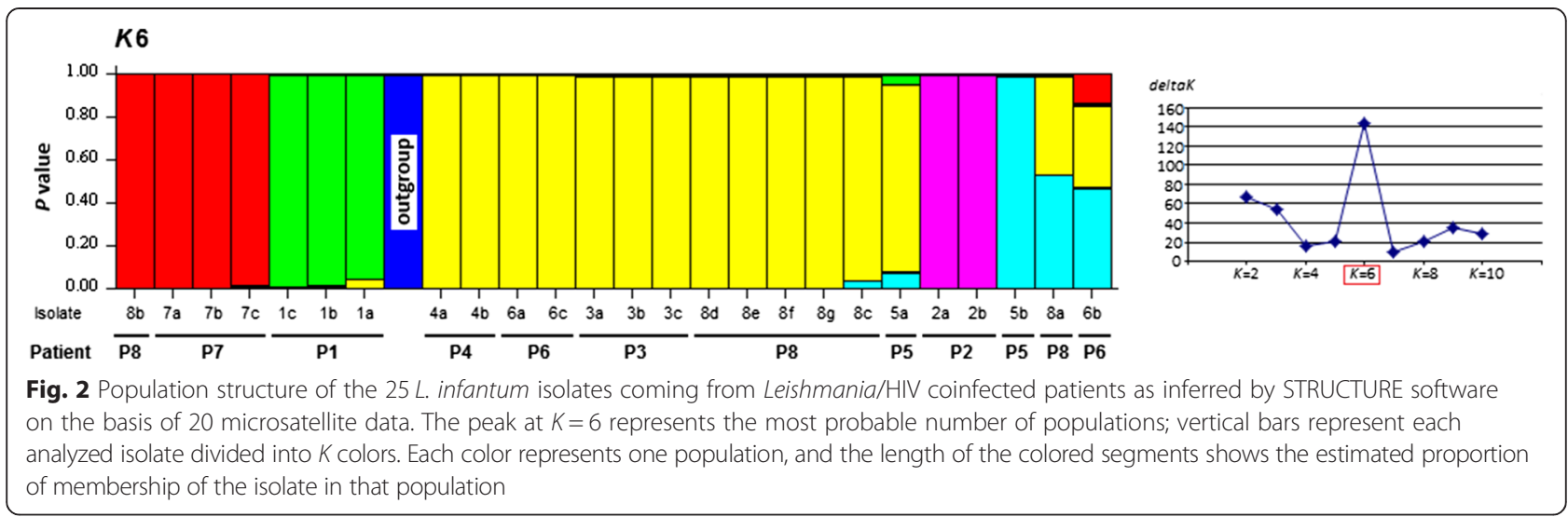

particular genotypes either, as previously described by other authors [26].

Our data indicated a high degree of heterogeneity and no predominant genotype in the isolates, but a more extensive study is required to assess the real variability and abundance of genotypes in immunocompromised patients in this area. The parasite heterogeneity observed in our patient set is in agreement with the genetic variability described in a previous MLMT study on Leishmania isolates from dogs and sand flies performed in a rural leishmaniasis-endemic area close to Barcelona (Priorat, Tarragona) [26]. Other MLMT studies performed on Leishmania parasites from different hosts, geographical regions and clinical forms have also found a low number of repeated multilocus genotypes $[25,26]$. A Portuguese study reported a generally low percentage $(12 \%)$ of repeated genotypes, although a higher percentage was found in coinfected patients [25], which was related to human-to-human transmission, notably associated with IVDU [27, 28]. The genotypic diversity registered in our study was 0.72 , which is higher than in other studies performed in Europe, for example, 0.55 in Greece [29] or 0.67 in Portugal [25]. However, any comparison between these results is limited by the variable number of isolates and loci analyzed.

Leishmania/HIV coinfection is characterized by frequent recurrences of clinical episodes. Previous studies with monitored L. infantum/HIV coinfected patients in our endemic area revealed the existence of a residual parasite load, using $\mathrm{GPCR}$ analysis after patient treatment, which has been related to the chronic course of the disease and subsequent recurrences of VL [30]. It is fair to assume that, in general, clinical recurrences may be related to relapses produced by the same parasite rather than reinfections. Nevertheless, detection by qPCR did not allow parasite characterization to differentiate between a relapse and reinfection [30]. In contrast, the use of MLMT analysis has proven useful in this respect and allowed to detect a high percentage of relapse cases among the clinical episodes of coinfected patients [4, 5, 25]. In our study, the phylogenetic reconstruction of our data by neighbor-joining (NJ) tree and STRUCTURE analysis was useful to visualize isolate distribution and genetic relationships.

In five patients (P1, P2, P3, P4 and P7), the NJ tree showed clear genetic links between the different isolates; either the genotype was exactly the same or slightly different. The isolates of two patients (P3 and P4) shared the same genotype, showing that these clinical episodes were due to the same Leishmania genotype. In these two patients, clinical episodes occurred 6 months after treatment and were not considered as a failure of treatment by clinicians. The close genetic similarity of isolates from the other three patients (P1, P2 and P7) may be due to the evolution of the Leishmania population in the patient under pharmacological and/or immunological pressure, and thus these cases could also be interpreted as a relapse. This suggests that in some cases the Leishmania population underwent an intrapatient evolution over time. In patients P2 and P7, this hypothesis is supported by the short time between the end of the treatment and the new clinical episode, which was considered by clinicians as a therapeutic failure, unlike the case of patient P1, with 8 and 12 months between clinical episodes.

The isolates of patients P5, P6 and P8, which showed a high dispersion in the NJ tree, suggested another hypothesis. The results obtained with STRUCTURE were globally in agreement with the genetic distance analysis, clearly indicating that isolates from these three patients correspond to different populations and some would be the result of mixing between populations, such as the $6 \mathrm{~b}$ and $8 \mathrm{a}$ samples. These differences cannot be explained only by reinfection events caused by sand fly transmission, since no transmission period occurred between some 
clinical episodes. A more probable explanation is that of a primo infection with multiple genotypes that were hidden by the culture. Nevertheless, the possible inoculation of different Leishmania genotypes by one or several sand flies does not rule out a reinfection by multiple sharing of contaminated syringes between IVDU.

The case of patient P8 is remarkable, with seven isolates, two from clinical episodes and five from non-clinical episodes, represented by 6 different and distant genotypes. Two genetically distant isolates were obtained with only a six-day interval, thus ruling out a reinfection, especially as the patient was hospitalized. Furthermore, this patient developed a second clinical episode, again with a different genotype. This high genetic variability suggests a mixed infection, with a genotype selection during the culture of the samples. Culture bias is a considerable inconvenience for the interpretation of results, as it is impossible to assess if one genotype is more responsible than another for the clinical episode. To avoid culture selection, it would be interesting to work directly with clinical samples, as other authors have done [31].

\section{Conclusion}

The use of multilocus microsatellite markers to analyze isolates taken from Leishmania/HIV coinfected patients allowed us to study the evolution of the infections. According to the results and clinical data, we had three different case types in our study: (i) two coinfected patients who relapsed, with the same genotypes in different clinical episodes (25\%); (ii) three coinfected patients who probably relapsed, with slightly different genotypes between clinical episodes due to the evolution of the parasite population within the patient (37.5\%), and (iii) three coinfected patients infected by several distant genotypes in the first clinical episode and a differential selection by the parasite culture performed for the study, or infected over time by non-vectorial transmission associated with IVDU (37.5\%). Due to the chronic nature of leishmaniasis in Leishmania/HIV coinfected patients and frequent clinical episode recurrences, close follow-up is required. Our study indicated a great MLMT variability between isolates from patients but, despite this, almost all clinical episodes could be interpreted as relapses.

The results obtained in this research have generated different hypotheses about Leishmania parasite behavior in coinfected patients. To explore these hypotheses in more depth, a broader study is required, using a higher number of stocks from coinfected patients monitored over time and from different geographical areas. This study also shows the value of parasite typing to provide new insights into the behavior of Leishmania parasites in human.

\section{Competing interests}

The authors declare that they have no competing interests.

\section{Authors' contributions}

MTP: designed the study, carried out the microsatellite studies, performed the statistical analysis and drafted the manuscript. $\mathrm{MH}$ : helped to carry out the microsatellite studies, coordinated and helped to perform the statistical analysis and helped to draft the manuscript. CR: conceived and designed the study, and helped to draft the manuscript. LM: participated in the microsatellite studies and the statistical analysis. ALB: helped to perform the statistical analysis and helped to draft the manuscript. ER: carried out the sampling. MP: helped to design the study and helped to draft the manuscript. RF: conceived and designed the study, coordinated and helped to carry out the microsatellite studies, helped to perform the statistical analysis and helped to draft the manuscript. All authors read and approved the final manuscript.

\section{Acknowledgments}

We thank the Unitat de Genòmica of the CCiTUB (Parc Científic, Universitat de Barcelona) for their collaboration on the study. We also thank Dr. M. Gállego and S. Tebar from Leishmania Cryobank of the Universitat de Barcelona, where strains used in this study were conserved. Data used in this work were (partly) produced through molecular genetic analysis technical facilities of the labex "Centre Méditerranéen de l'Environnement et de la Biodiversité", France.

This work is part of a research study supported by SGR1241 Generalitat de Catalunya.

\section{Author details}

${ }^{1}$ Laboratory of Parasitology, Faculty of Pharmacy, Universitat de Barcelona, Avda Joan XXIII s/n, 08028 Barcelona, Spain. ${ }^{2}$ MIVEGEC, UMR IRD 224-CNRS 5290-Université de Montpellier, Montpellier, France. ${ }^{3}$ Infectious Diseases Department and Microbiology Department, Hospital Universitari Vall d'Hebron, Universitat Autònoma de Barcelona, Barcelona, Spain.

Received: 10 February 2015 Accepted: 6 July 2015

Published online: 22 July 2015

\section{References}

1. Alvar J, Aparicio P, Aseffa A, Den Boer M, Cañavate C, Dedet JP, et al. The relationship between leishmaniasis and AIDS: the second 10 years. Clin Microbiol Rev. 2008;21:334-59.

2. WHO. Control of the Leishmaniases. In: WHO Technical Report Series. Geneva: World Health Organization; 2010. p. 949.

3. Morales MA, Cruz I, Rubio JM, Chicharro C, Cañavate C, Laguna F, et al. Relapses versus reinfections in patients coinfected with Leishmania infantum and human immunodeficiency virus type 1. J Infect Dis. 2002;185:1533-7.

4. Kuhls K, Chicharro C, Cañavate C, Cortés S, Campino L, Haralambous C, et al. Differentiation and gene flow among European populations of Leishmania infantum MON-1. PLoS Negl Trop Dis. 2008;2, e261.

5. Gelanew T, Kuhls K, Hurissa Z, Weldegebreal T, Hailu W, Kassahun A, et al. Inference of population structure of Leishmania donovani strains isolated from different Ethiopian visceral leishmaniasis endemic areas. PloS Negl Trop Dis. 2010;4, e889.

6. Pratlong F, Dereure J, Deniau M, Marty P, Faraut-Gambarelli F, Dedet JP. Enzymatic polymorphism during Leishmania/HIV co-infection: a study of 381 Leishmania strains received between 1986 and 2000 at the international cryobank in Montpellier France. Ann Trop Med Parasitol. 2003;97:47-56.

7. Gállego M, Pratlong F, Riera C, Muñoz C, Ribera E, Fisa R, et al. Isoenzymatic identification of Leishmania isolates from repeated clinical human leishmaniasis episodes in Catalonia (Spain). Trans R Soc Trop Med Hyg. 2002;96:45-7.

8. Chicharro C, Jiménez Ml, Alvar J. Iso-enzymatic variability of Leishmania infantum in Spain. Ann Trop Med Parasitol. 2003;97:57-64.

9. Martín-Sánchez J, Gramiccia M, Di Muccio T, Ludovisi A, Morillas-Márquez F. Isoenzymatic polymorphism of Leishmania infantum in southern Spain. Trans R Soc Trop Med Hyg. 2004;98:228-32.

10. Toth G, Gaspari Z, Jurka J. Microsatellites in different eukaryotic genomes: survey and analysis. Genome Res. 2000;10:967-81.

11. Ochsenreither S, Kuhls K, Schaar M, Presber W, Schönian G. Multilocus microsatellite typing as a new tool for discrimination of Leishmania infantum MON-1 strains. J Clin Microbiol. 2006;44:495-503. 
12. Schönian G, Kuhls K, Mauricio IL. Molecular approaches for a better understanding of the epidemiology and population genetics of Leishmania. Parasitology. 2011;138:405-25.

13. Piarroux R, Azaiez R, Lossi AM, Reynier P, Muscatelli F, Gambarelli F, et al. Isolation and characterization of a repetitive DNA sequence from Leishmania infantum: development of a visceral leishmaniasis polymerase chain reaction. Am J Trop Med Hyg. 1993;49:364-9.

14. Fisa R, Riera C, Ribera E, Gállego M, Portús M. A nested polymerase chain reaction for diagnosis and follow-up of human visceral leishmaniasis patients using blood samples. Trans R Soc Trop Med Hyg. 2002;96:191-4.

15. Goudet J, Perrin N, Waser P. Tests for sex-biased dispersal using bi-parentally inherited genetic markers. Mol Ecol. 2002;11:1103-14.

16. Goudet J. FSTAT (Version 1.2): a computer program to calculate F-statistics. J Hered. 1995;86:485-6.

17. Nei M, Chesser RK. Estimation of fixation indices and gene diversities. Ann Hum Genet. 1983;47:253-9.

18. Saitou N, Nei M. The neighbor-joining method: a new method for reconstructing phylogenetic trees. Mol Biol Evol. 1987;4:406-25.

19. Chevenet F, Brun C, Bañuls AL, Jacq B, Christen R. TreeDyn: towards dynamic graphics and annotations for analyses of trees. BMC Bioinformatics. 2006;7:439.

20. Pritchard JK, Stephens M, Donnelly P. Inference of population structure using multilocus genotype data. Genetics. 2000;155:945-59.

21. Evanno G, Regnaut S, Goudet J. Detecting the number of clusters of individuals using the software STRUCTURE: a simulation study. Mol Ecol. 2005; 14:2611-20.

22. Garnier S, Alibert P, Audiot P, Prieur B, Rasplus JY. Isolation by distance and sharp discontinuities in gene frequencies: implications for the phylogeography of an alpine insect species, Carabussolieri. Mol Ecol. 2004;13:1883-97.

23. Chicharro C, Alvar J. Lower trypanosomatids in HIV/AIDS patients. Ann Trop Med Parasitol. 2003;97:75-8.

24. Hide M, Marion E, Pomares C, Fisa R, Marty P, Bañuls AL. Parasitic genotypes appear to differ in leishmaniasis patients compared with asymptomatic related carriers. Int J Parasitol. 2013;43:389-97.

25. Cortés S, Maurício IL, Kuhls K, Nunes M, Lopes C, Marcos M, et al. Genetic diversity evaluation on Portuguese Leishmania infantum strains by multilocus microsatellite typing. Infect Genet Evol. 2014;26:20-31.

26. Montoya L, Gállego M, Gavignet B, Piarroux R, Rioux JA, Portús M, et al. Application of microsatellite genotyping to the study of a restricted Leishmania infantum focus: different genotype compositions in isolates from dogs and sand flies. Am J Trop Med Hyg. 2007;76:888-95.

27. Campino L, Santos-Gomes G, Pratlong F, Dedet JP, Abranches P. The isolation of Leishmania donovani MON-18, from an AIDS patient in Portugal: possible needle transmission. Parasite. 1994;1:391-2.

28. Cruz I, Morales MA, Noguer I, Rodríguez A, Alvar J. Leishmania in discarded syringes from intravenous drug users. Lancet. 2002;359:1124-5.

29. Gouzelou E, Haralambous C, Antoniou M, Christodoulou V, Martinković F, Živičnjak T, et al. Genetic diversity and structure in Leishmania infantum populations from southeastern Europe revealed by microsatellite analysis. Parasit Vectors. 2013;5:342.

30. Molina I, Fisa R, Riera C, Falcó V, Elizalde A, Salvador F, et al. Ultrasensitive real-time PCR for the clinical management of visceral leishmaniasis in HIV-Infected patients. Am J Trop Med Hyg. 2013;89:105-10.

31. Motoie G, Ferreira GE, Cupolillo E, Canavez F, Pereira-Chioccola VL. Spatial distribution and population genetics of Leishmania infantum genotypes in São Paulo State, Brazil, employing multilocus microsatellite typing directly in dog infected tissues. Infect Genet Evol. 2013;18:48-59.

32. Bulle B, Millon L, Bart JM, Gállego M, Gambarelli F, Portús M, et al. Practical approach for typing strains of Leishmania infantum by microsatellite analysis. J Clin Microbiol. 2002;40:3391-7.

33. Jamjoom MB, Ashford RW, Bates PA, Kemp SJ, Noyes HA. Towards a standard battery of microsatellite markers for the analysis of the Leishmania donovani complex. Ann Trop Med Parasitol. 2002;96:265-70.

34. Rossi V, Wincker P, Ravel C, Blaineau C, Pages M, Bastien P. Structural organization of microsatellite families in the Leishmania genome and polymorphisms at two (CA)n loci. Mol Biochem Parasitol. 1994;65:271-82. 